Revista de Matemática: Teoría y Aplicaciones 2(1): 27-37 (1995)

\title{
LOBACHEVSKI DESCUBRIDOR DE LA GEOMETRÍA HIPERBÓLICA
}

\author{
Juan Bautista Boza Cordero ${ }^{1}$
}

\begin{abstract}
Resumen
En ocasión del segundo centenario del nacimiento del ilustre matemático ruso N.I. Lobachevski (1792-1856), se presenta una visión global de su trabajo geométrico, que culminó con el descubrimiento de la geometría hiperbólica. Se analiza el rol del V Postulado en la geometría euclídea y los primeros intentos por demostrarlo, realizados hasta el siglo XIX. Se exponen las principales ideas de la solución dada por Lobachevski al "Problema de las Paralelas", es decir, los fundamentos de su nueva geometría. Se examina el impacto de la misma en las discusiones acerca de qué es el espacio y qué la geometría. Finalmente se hace referencia a la influencia de la geometría hiperbólica en la física.
\end{abstract}

\begin{abstract}
We offer an overview of the geometric studies of the russian mathematician N.I. Lobachevski (1792-1856), which culminated with the discovery of the hyperbolic geometry. We analize the role of the $\mathrm{V}$ Postulate in the euclidean geometry and the ways some mathematicians tried to prove it. We also examine the foundations of the new geometry and some of its relations with physics and philosophy.
\end{abstract}

\section{Introducción}

El libro los Elementos de Euclides (finales del siglo IV y principios del siglo III a.n.e.) constituyó un esfuerzo por fundamentar sólidamente los conocimientos matemáticos de la época, y en lo referente a la geometría, se mantuvo por más de veinte siglos como la referencia obligada, y prácticamente indiscutible, en todo el mundo occidental.

Una de las características que los griegos le atribuían a la geometría era la de ser un cuerpo de conocimientos sobre el mundo material, aquél donde se desarrollan los fenómenos físicos. El interés de los griegos por organizar y sistematizar la ciencia geométrica los llevó a presentarla como una ciencia deductiva, de ahí que realizaran ingentes esfuerzos por reducir al máximo el número de aquellas afirmaciones elementales, a partir de las cuales pudieran derivarse lógicamente las demás proposiciones. Resultado de esta empresa fue el sistema axiomático de la geometría hoy llamada euclídea y expuesto en los Elementos.

\footnotetext{
${ }^{1}$ Escuela de Matemática, Universidad de Costa Rica
} 
Sobre estas bases se desarrolló la geometría hasta principios del siglo XIX, cuando los descubrimientos de LOBACHEVSKI y Bolyai le quitaron a la geometría euclídea el supuesto carácter de ciencia universal e inmutable del espacio. A partir de entonces se supo que no existe sólo una geometría, y que las geometrías euclídea e hiperbólica, aunque basadas en hipótesis antagónicas, son igualmente posibles y ninguna es "más verdadera" que la otra.

Estos descubrimientos conmovieron al mundo matemático durante gran parte del siglo pasado, pues mostraron que el concepto de espacio que se venía manejando era innecesariamente restringido, lo cual llevó de manera natural a replantearse el carácter mismo de la geometría y de sus objetos de estudio. El surgimiento de las geometrías no euclídeas también repercutió positivamente en ciencias como la física y la astronomía y en la filosofía.

Aprovechamos que este año se cumple el segundo centenario del nacimiento de NiKOLAI IVÁNOVICH LOBACHEVSKI $(1792-1856)^{2}$, a quien la mayoría de los autores le atribuyen los mayores méritos en el descubrimiento y desarrollo de las geometrías no euclídeas, para exponer resumidamente sus principales ideas y el impacto de las mismas en campos afines.

\section{La teoría de las paralelas}

La exposición de la geometría en los Elementos de EuCLIDES comienza por enunciar un número reducido de "nociones comunes", algunas reglas de construcción de figuras y cinco postulados, a partir de los cuales se pretende deducir las demás proposiciones. La idea es que estos primeros enunciados sean lo bastante sencillos e intuitivos, para que el lector los acepte como verdaderos, y lograr entonces que las proposiciones demostradas, utilizando de manera rigurosa e impecable las reglas de la lógica, se impongan definitivamente.

Utilizando únicamente los primeros cuatro postulados, junto con las reglas de construcción y las nociones comunes, EuCLIDEs demostró 27 proposiciones referentes a los triángulos (su construcción; relaciones entre los lados y los ángulos de un mismo triángulo o de dos triángulos distintos), así como sobre los ángulos adyacentes y los ángulos opuestos por el vértice.

La polémica alrededor de la teoría de las paralelas se inicia a partir de la proposición 27 , que puede enunciarse como sigue ([3, p.128]):

Proposición 27. Considere dos rectas $l_{1} y l_{2}$ en un mismo plano, que son cortadas por una tercera $l_{3}$, de manera que se cumple una (y, por tanto, todas) de las relaciones (véase Figura 1):

(1) $c=e$ ó $d=f$,

(2) $a=g$ ó $b=h$,

(3) $a=e$ ó $b=f$ ó $c=g$ ó $d=h$,

(4) alguna de las sumas $c+f, d+e, a+h$ ó $b+g$ es igual a dos rectos.

Entonces $l_{1}$ y $l_{2}$ son paralelas, es decir, no se cortan

\footnotetext{
${ }^{2}$ Este artículo fue originalmente escrito en 1992 y propuesto para su publicación en la desaparecida revista Ciencias Matemáticas
} 


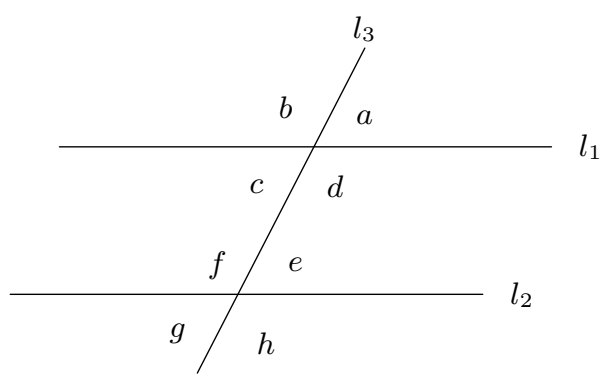

Figura 1. (Proposición 27)

El recíproco de la proposición 27 llamó la atención desde antes de la época de EuCLíDEs; y como se intuía que tenía que ser verdadero, algunos geómetras emprendieron la tarea de demostrarlo (Proclo, Posidonio, cfr. [1, p.2]). Pero Euclides no se hizo mayor problema, y de hecho tomó el enunciado de dicho recíproco y lo incorporó como su $\mathrm{V}$ postulado, a continuación de la proposición 27.

V Postulado. Si en un mismo plano una recta corta a otras dos, y la suma de los ángulos interiores del mismo lado es menor que dos rectos, entonces esas rectas, al prolongarlas indefinidamente, se cortan del lado en que la suma de tales ángulos es menor que dos rectos. (Figura 2.)

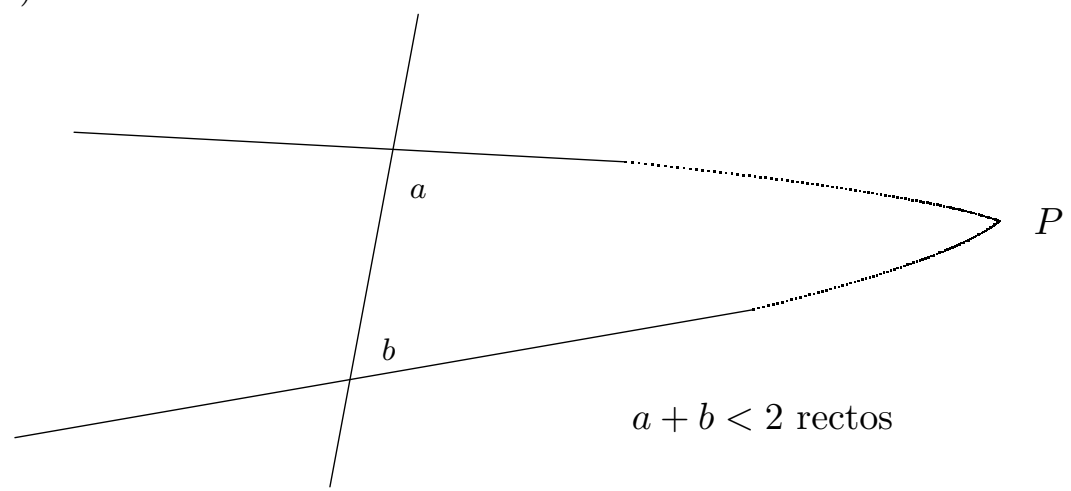

Figura 2. (V Postulado)

El $\mathrm{V}$ postulado fue ubicado por Euclides en lugar aparte, no junto a los primeros cuatro, sino después de la proposición 27. Además, se diferencia de ellos por cuanto su enunciado no aparece tan simple y más bien invita a ser demostrado.

La geometría euclídea se mantuvo sin ser cuestionada a fondo durante varios siglos, y era considerada como la única verdadera teoría del espacio. Pero en el Renacimiento, el interés por comprender la obra de EuCliDEs hizo que se pusiera a discusión nuevamente, y con mayor ímpetu, "el problema de las paralelas". Característico de todos estos esfuerzos, que se prolongaron hasta principios del siglo XIX, fue la certidumbre de que el V postulado era cierto y había que encontrarle una demostración. 
A los intentos por demostrar el V postulado están asociados los nombres de PLAYFAIR, Wallis, Saccheri, Lambert, Legendre, Gauss, e incluso Bolyai y Lobachevski. Hoy se ha establecido que los tres últimos autores se percataron, de manera totalmente independiente, de que la empresa era imposible, y debía buscarse una solución distinta y novedosa. ([7, p.123]). Por diversas razones Gauss no se decidió a profundizar en sus ideas, en tanto Bolyai y Lobachevski, cada uno por su lado y sin comunicarse entre sí, desarrollaron una nueva geometría, en la cual el $\mathrm{V}$ postulado no es válido, y más bien se cumple la negación del mismo. Los trabajos de los dos últimos autores guardan una semejanza impresionante; de hecho, ambos descubrieron la geometría hiperbólica. Los mayores méritos se asignan normalmente a LOBACHEVSKI porque fue el primero que publicó sus resultados (1826) y logró presentar su geometría, luego de varios años de trabajo, como una teoría con solidez interna, que además tenía cosas importantes que decir a la mecánica y la astronomía.

\section{Las “demostraciones" del V Postulado}

Hoy sabemos con certeza que no es posible demostrar el V postulado a partir de los anteriores ([1, p.177]) y ello es así porque existen modelos, tanto de geometría euclídea (que acepta dicho postulado), como para la geometría hiperbólica (que acepta la negación del mismo). Tales modelos garantizan que cada uno de estos sistemas axiomáticos es consistente, es decir, en ninguno es posible encontrar una sola proposición, tal que ella y su negación sean verdaderas.

La historia de los esfuerzos por demostrar el V postulado es rica y abundante, además de estar bien documentada ([1]). Nos vamos a referir brevemente a los intentos más sobresalientes hechos a partir del Renacimiento. Muchos de los autores pensaron toda su vida que, efectivamente, habían logrado demostrar el V postulado; otros, no estuvieron tan convencidos. En todo caso, lo que apreciamos en la actualidad es que casi en todas las argumentaciones se apelaba en cierto momento a un enunciado, que se daba por cierto, pero que en realidad era equivalente al $\mathrm{V}$ postulado, lo cual obviamente invalidaba el razonamiento.

Entre todos los esfuerzos, destaca el de SACCHERI (1667-1733), quien se propuso demostrar que si se aceptaban los cuatro primeros postulados y la negación del quinto, entonces existía una proposición, tal que ella y su negación eran simultáneamente verdaderas. Según SACCHERI, tomando en cuenta que nadie dudaba de los primeros cuatro postulados ni de sus primeras consecuencias (las 27 proposiciones), esto pondría en evidencia la falsedad de la negación del $\mathrm{V}$ postulado, y con ello, la verdad del quinto postulado.

SACCHERI empezó entonces por hallar una formulación adecuada a la negación del V postulado, y por este camino examinó detenidamente un tipo de cuadrilátero, obtenido al levantar desde puntos $A$ y $B$ sendas perpendiculares, para luego tomar puntos $A^{\prime}, B^{\prime}$ en dichas rectas, con $A A^{\prime}=B B^{\prime}$ (Figura 3). 


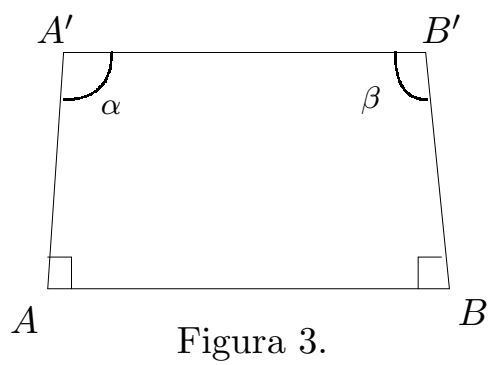

Fácilmente demostró que entonces $\alpha=\beta$. Enseguida observó que se dan tres posibilidades: (1) $\alpha=\beta=90^{0}$; (2) $\alpha=\beta<90^{0}$; (3) $\alpha=\beta>90^{\circ}$; y lo importante es que (2) y (3) niegan el $\mathrm{V}$ postulado. Con un poco más de trabajo logró demostrar que (1) implica que la suma de los ángulos internos de cualquier triángulo es igual a dos rectos; que (2) implica que tal suma es menor que dos rectos; y que (3) implica que dicha suma es mayor que dos rectos. Así mismo, demostró que (1) es equivalente al V postulado. Por consiguiente, continúa su razonamiento, para demostrar el $\mathrm{V}$ postulado es suficiente demostrar que las hipótesis (2) y (3) conducen a contradicciones.

La hipótesis (3) la descarta él con una argumentación clara y convicente ([7, p.63]). Al examen de la hipótesis (2) dedica SACCHERI unas 80 páginas, en donde considera cierto tipo de rectas que se aproximan asintóticamente y que dan la impresión de que se cortan en un punto situado en el infinito, punto en el cual él es capaz de construir una perpendicular común. SACCHERI cierra entonces su refutación a la hipótesis (2), diciendo que la conclusión por él obtenida es contraria a la naturaleza de las rectas.

Aunque hoy la refutación de SACCHERI a la hipótesis (2) es a todas luces insuficiente, algunas de las ideas apuntaban certeramente hacia una nueva geometría, de lo cual él mismo nunca se percató.

J. WALlis (1616-1703) dio a conocer en 1663 una "demostración" del V postulado que descansaba en la posibilidad de construir, dado un triángulo y una razón, otro triángulo semejante al primero precisamente por la razón dada. De lo que este autor nunca se dio cuenta es que esta suposición es equivalente a lo que se proponía demostrar, es decir, al V postulado.

Como mencionamos hace unos momentos, el $\mathrm{V}$ postulado es equivalente a que la suma de los ángulos internos de un triángulo es dos rectos. LEGENDRE (1752-1833), conocedor de este hecho, se propuso establecer la verdad del V postulado, demostrando, sin hacer uso del mismo, que la suma de los ángulos interiores de un triángulo es dos rectos. No vamos a examinar su argumentación, pero en ella él da por un hecho que por todo punto interior de un ángulo siempre es posible trazar una recta que corta los dos lados del ángulo en puntos distintos del vértice. Se demostró posteriormente que este enunciado es equivalente al V postulado, con lo cual su intento perdió fuerza.

\section{Hacia una nueva geometría}

A principios del siglo XIX se empieza a dar un cambio en la actitud con respecto al $\mathrm{V}$ postulado, lo que que no se manifestó de manera repentina ni generalizada, y se debió más bien a un cambio gradual en algunos geómetras, que de manera aislada se fueron 
convenciendo de la imposibilidad de demostrar el V postulado. Es claro que llegar a esta posición significaba empezar a romper con una tradición muy arraigada, de más de veinte siglos. Y persistir en el empeño de sacar todas las consecuencias de negar el $\mathrm{V}$ postulado, requería de una fuerte convicción y la valentía necesaria para oponerse a las corrientes dominantes en el ámbito académico.

Algunos pocos autores atribuyen a GaUss la prioridad en el descubrimiento de las nuevas geometrías; pero estudios recientes de su correspondencia, tienden a ubicar su contribución en un lugar más bien modesto. Aunque está claro que él se ocupó del problema de las paralelas desde finales del siglo XVIII, y que de manera privada hizo comentarios a algunos de sus allegados, es lo cierto que ello no tuvo mayor trascendencia, quedó oculto para la comunidad matemática ([7, p.123]).

Tanto LoBACHevski como Bolya tomaron contacto con el problema de las paralelas dentro de la corriente que pretendía demostrar el $\mathrm{V}$ postulado, y sólo después de un tiempo desistieron de esta tarea, para emprender de manera independiente la formidable aventura de crear una geometría basada en el hecho de que por un punto exterior a una recta, y en el mismo plano, pasa más de una paralela a la original (enunciado equivalente al $\mathrm{V}$ postulado, según lo demostró Playfair).

Característico de los nuevos enfoques fueron las consideraciones trigonométricas, en contraposición con los tratamientos clásicos. Empezaremos por hacer breve mención de algunas investigaciones realizadas por autores alemanes, que hoy se ven como pudieron llegar a ser los primeros pasos en la nueva geometría, pero que se quedaron como logros aislados.

En 1818 SchweIKART (1780-1859) le comunicó a GAUSS sus investigaciones acerca de la geometría astral, en la cual las paralelas no son únicas. El demostró que en tal geometría existe un tamaño máximo para la altura de un triángulo recto. En su respuesta Gauss le indica que ya él ha desarrollado esta nueva geometría, y que está en capacidad de resolver todos los problemas de ella, una vez conocido el valor de la altura máxima estudiada por SchweIKART, a la vez que le da a conocer una fórmula sencilla para el área máxima. Hasta hoy no se ha podido documentar fehacientemente el avance anunciado por GAuss.

TAURINUs (1794-1874), quien mantuvo contacto con SCHWEIKART y en alguna medida con Gauss, dedujo una fórmula que relaciona el ángulo de paralelismo con la correspondiente altura en la cual aparece la altura máxima $c$ de SCHWEIKART (Figura 4).

$$
\cosh \frac{a}{k}=\frac{1}{\operatorname{sen} A} \quad \text { donde } k=\frac{c}{\log (1+\sqrt{2})}
$$

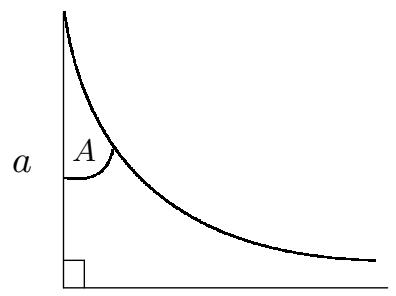

Figura 4.

El enfoque de TAURINUs tiene la peculariedad de estar basado en consideraciones trigonométricas; él combinó fórmulas de la trigonometría esférica con las funciones hiperbólicas introducidas 
por EULER y sistemáticamente estudiadas por LAMBERT en 1768.

\section{$5 \quad$ La geometría hiperbólica}

Una vez que LoBACHEVSKI admite que por un punto exterior a una recta, y en el plano por ella determinado, existe más de una recta paralela a la recta considerada, él demuestra que el número de tales paralelas es infinito.

Si $P$ es un punto fuera de la recta $A B$, y se considera la perpendicular $P Q$ a $A B$ por $P$, entonces la perpendicular $C D$ por $P$ a la recta $P Q$, es paralela a $A B$ (Figura 5 ).

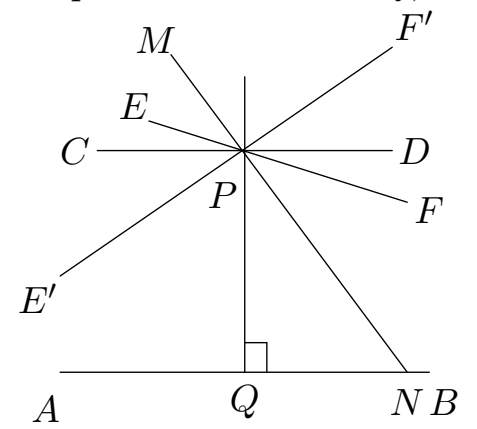

Figura 5.

El haz de todas las rectas por el punto $P$ consta de aquéllas que cortan a $A B$ (como la recta $M N)$ y de las que no cortan a $A B$. Como además de $C D$ existe otra paralela por $P$, LoBACHEVSKI logra determinar dos rectas limite $E F$ y $E^{\prime} F^{\prime}$ que no cortan $A B$, y tales que toda recta por $P$ situada en el ángulo $E^{\prime} P F$ corta a $A B$; y, por el contrario, ninguna recta situada en uno de los ángulos $E P E^{\prime}$ o $F^{\prime} P F$, corta a $A B$.

El llama a estas rectas límite las paralelas a $A B$ por $P$. Demuestra que los ángulos $Q P E^{\prime}$ y $Q P F$ son iguales, y este valor común lo llama ángulo de paralelismo por $P$. También demuestra que las paralelas $A B$ y $P F$ se aproximan asintóticamente "en el sentido del paralelismo", (es decir, al prolongarlas hacia la derecha), como si se cortaran en un punto situado en el infinito; en el sentido opuesto, estas rectas se alejan indefinidamente. Las rectas contenidas en los ángulos $E P E^{\prime}$ y $F^{\prime} P F$ las llama divergentes (de $A B$ ); él demuestra que dos rectas divergentes cualesquiera poseen una perpendicular común, que mide la distancia mínima entre ellas, y a partir de la cual divergen y se alejan una de la otra indefinidamente en ambas direcciones (Figura 6).

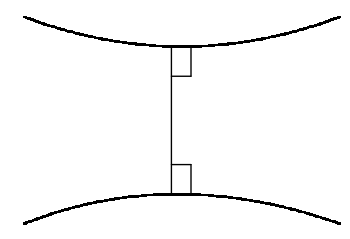

Figura 6.

Si $A B$ es una recta y se considera la perpendicular a ella desde un punto $P$, el ángulo de paralelismo $Q P F$ por el punto $P$ depende de la altura $P Q$. En efecto, si se toma un punto $P^{\prime}$ situado por encima de $P$, la recta $P^{\prime} T$, que forma con $P^{\prime} Q$ un ángulo igual $Q P F$, y la recta $P F$, son paralelas euclidianas que, por lo tanto, divergen. Entonces la recta $P^{\prime} F^{\prime}$, 
paralela a $A B$ por $P^{\prime}$, está más cerca de $P^{\prime} Q$ que la recta $P^{\prime} T$, lo cual evidencia que el ángulo de paralelismo es una función $\pi(x)$ de la distancia $x$ del punto $P$ a la recta $A B$ (Figura 7). El ángulo de paralelismo es agudo, y disminuye al aumentar la distancia del punto $P$ a la recta $A B$. También demostró LoBACHEvski que el ángulo de paralelismo tiende al ángulo recto conforme la distancia del punto a la recta $A B$ disminuye.

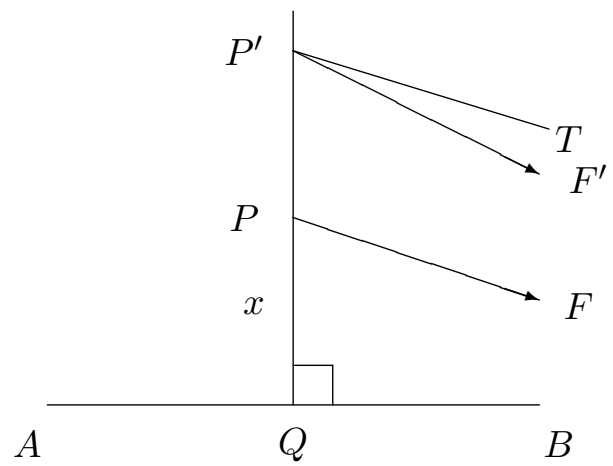

Figura 7.

Gran interés prestó Lobachevski a la tarea de determinar explícitamente la función $\Pi(x)$, en donde $\Pi(x)$ es el ángulo de paralelismo correspondiente a la altura $x$ del punto $P$ sobre el punto $Q$, para finalmente obtener la relación

$$
\operatorname{cotg} \frac{\Pi(x)}{2}=q^{x}
$$

en donde $q$ es una constante mayor que 1 ([3, p.158]); esta relación se conoce como la "ecuación fundamental" de la geometría hiperbólica.

Previamente había obtenido las siguientes igualdades para un triángulo rectángulo $A B C$ con catetos $a, b$ :

$$
\begin{aligned}
\operatorname{cotg} \Pi(a) & =\operatorname{cotg} \Pi(c) \operatorname{sen} A \\
\operatorname{cotg} \Pi(b) & =\operatorname{cotg} \Pi(c) \operatorname{sen} B \\
\operatorname{sen} \Pi(a) \operatorname{sen} \Pi(b) & =\operatorname{sen} \Pi(c)
\end{aligned}
$$

para las funciones trigonométricas ordinarias ([3, p.157]). 


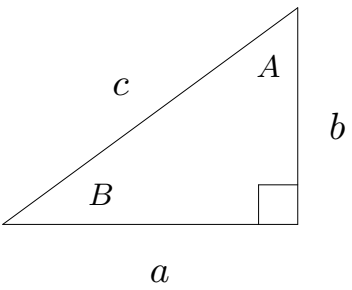

Figura 8.

De manera que en ese momento ya pudo "resolver" el triángulo $A B C$, es decir, hallar, por ejemplo, un cateto si se conocen la hipotenusa y el ángulo opuesto.

Las relaciones (1) le permitieron establecer conexiones importantes con las recién introducidas funciones hiperbólicas. Para ello definió una nueva constante $k$, mediante

$$
q=e^{1 / k}, \quad \text { es decir } k=\frac{1}{\ln q}
$$

de modo que pudo escribir

$$
\operatorname{cotg} \frac{\Pi(x)}{2}=e^{x / k}
$$

De aquí obtuvo

$$
\begin{aligned}
\operatorname{cotg} \Pi(x) & =\frac{e^{x / k}-e^{-x / k}}{2} \\
\csc \Pi(x) & =\frac{e^{x / k}+e^{-x / k}}{2},
\end{aligned}
$$

lo cual permite escribir las relaciones (1) así ([3, p.190]):

$$
\begin{aligned}
e^{a / k}-e^{-a / k} & =\left(e^{c / k}-e^{-c / k}\right) \operatorname{sen} A \\
e^{b / k}-e^{-b / k} & =\left(e^{c / k}-e^{-c / k}\right) \operatorname{sen} B \\
e^{c / k}-e^{-c / k} & =\frac{1}{2}\left(e^{a / k}+e^{-a / k}\right)\left(e^{b / k}+e^{-b / k}\right) .
\end{aligned}
$$

La primera ecuación, por ejemplo, se escribe de manera más concisa como

$$
\operatorname{senh}(a / k)=\operatorname{senh}(c / k) \operatorname{sen} A .
$$

Y es debido a estas relaciones con las funciones hiperbólicas, que a la nueva geometría se le conoció como la "geometría hiperbólica".

\section{Breve nota histórica}

LOBACHEVSKI desarrolló toda su actividad científica en la Universidad de Kazán, de la cual fue alumno y luego profesor y rector. Allí expuso por vez primera los fundamentos de su nueva geometría, en conferencia dictada en la Facultad de Físico-Matemáticas, en 1826. De esta conferencia no se conserva copia; pero en 1929 publicó en Kazán su monografía Acerca de los principios de la geometría, escrita en ruso y que no fue bien recibida en su 
medio, probablemente por lo novedoso y audaz de sus planteamientos, sumado a recelos e intrigas de que era objeto ([3]).

Interesado en ampliar su auditorio, publicó en 1840 sus Investigaciones Geométricas en alemán, y por una editorial alemana. Ambas obras llegaron a manos de Gauss, considerado ya el matemático más destacado de la época, y quien se referió en términos elogiosos de su autor, si bien en privado.

LOBACHEVSKI se desenvolvió siempre en un medio académico adverso, tanto en lo científico como hacia su labor administrativa de rector. A pesar de que su nombre ha pasado a la historia, en vida no recibió el reconocimiento por su obra. No fue sino después de unos treinta años de publicados, que sus escritos merecieron la atención de matemáticos de su país y de Europa.

A lo largo de toda su vida, su pasión fue la geometría, siempre preocupado, hasta sus últimos días, por divulgar sus ideas y mejorar su presentación. Es digno de admiración su decisión por desarrollar su sistema geométrico, a pesar de la valoración tan negativa que del mismo se hizo desde un principio.

LOBACHEVSKI fue muy consciente de que luchaba contra una tradición milenaria, y vale la pena reflexionar sobre los motivos que lo mantuvieron activo en esta lid. Su revolucionaria concepción del espacio y de la misma geometría chocaron frontalmente con las ideas prevalecientes. Esa fuerza para seguir adelante en sus investigaciones provenía, en primer lugar, de su capacidad para intuir el espacio que él mismo estaba descubriendo. Pero también contaba con criterios más seguros para darse cuenta de que, a pesar de los riesgos asumidos, era bastante probable que estuviera trabajando en firme. A estas conclusiones lo conducían, por ejemplo, las conexiones que estableció con las funciones trigonométricas hiperbólicas, relaciones que él consideraba no podían ser casuales. En el mismo sentido valoraba los cálculos de algunas integrales definidas, realizados por medios puramente geométricos, y que luego comprobaba analíticamente. Pero el mismo LobAchevski consideraba que la validez de su geometría no podía establecerse por medios como los mencionados. Su opinión era que para decidir cuál geometría es la verdadera, había que remitirse a la práctica y entonces determinar, por ejemplo mediante mediciones astronómicas, si la suma de los ángulos internos de un triángulo es igual o menor que dos rectos. El mismo buscó esa comprobación, pero no llegó a conclusiones satisfactorias.

\section{La cuestión de la validez}

En un principio se consideró que la aparición de la geometría hiperbólica traía consigo la inevitable tarea de decidir cuál de las geometrías era "la verdadera", lo cual se entendía como determinar si el espacio material es euclídeo o hiperbólico. Con ello se estaba buscando una respuesta a un problema matemático fuera de la matemática misma. La validez de un sistema geométrico se refiere a que de los axiomas que lo fundamentan, no pueda deducirse ninguna proposición contradictoria. Un sistema axiomático que está libre de contradicciones se llama consistente.

La consistencia de la geometría hiperbólica se estableció a lo largo de un proceso complejo. La idea básica es que para demostrar la consistencia de un sistema axiomático, basta

encontrar un modelo para el mismo, es decir, un sistema de objetos concretos que satisfagan 
dichos axiomas. En la base está la consideración de que lo real, lo que existe, no puede ser contradictorio.

El primer paso fue dado por BeLTRAMI (1835-1900), quien a partir de la trigonometría hiperbólica establecida por LoBACHEvski, demostró la "consistencia relativa" de las geometrías euclídea e hiperbólica. En efecto, él encontró un modelo para cada una de ellas en la otra, con lo cual estableció que cualquiera de ellas es consistente en la medida en que la otra lo es.

En particular, él estableció que la geometría de una superficie de curvatura constante negativa (una seudoesfera), puede considerarse como una interpretación concreta de la geometría hiperbólica. Pero subsistía una limitación, y es que en la seudoesfera no puede modelarse todo el espacio hiperbólico; más aún, como lo demostró HiLBERT, en el espacio ordinario no existen superficies que satisfagan en su totalidad las propiedades de la geometría hiperbólica ([1, Cap.V]).

Cuando se establecieron las limitaciones de los resultados de Beltrami, algunos de los matemáticos más destacados de la época se ocuparon del problema, que fue resuelto en sentido positivo y de manera definitiva, hacia finales del siglo pasado, gracias al concurso de Klein, Poincaré y Hilbert. Se supo entonces que ambas geometrías son consistentes y que ninguna es "más verdadera" que la otra ([1, Apéndice V]).

\section{Impacto en campos afines}

La concepción filosófica predominante a principios del siglo pasado era la kantiana, que armonizaba perfectamente con la geometría euclídea. Para KANT, en la geometría subyace conocimiento verdadero acerca del espacio ordinario. Este es un conocimiento a priori, independiente de cualquier experiencia particular, ejemplo de tal conocimiento son los axiomas de la geometría euclídea, considerados como verdades evidentes para cualquier persona de manera inmediata.

Para KANT nuestras representaciones del espacio y el tiempo no sólo son inherentes a nuestra conciencia, sino que constituyen las formas absolutamente necesarias del pensamiento, fuera de las cuales éste es imposible ([3, p.264]).

LOBACHEVSKI sostenía una concepción del espacio opuesta a la de KANT ([1, p.9293]). En efecto, él consideraba que las nociones fundamentales de la geometría se adquieren por los sentidos, y no debe recurrirse a ninguna noción innata ([3, p.264]). De aquí su insistencia de que sólo la experiencia puede decidir qué geometría tiene lugar en realidad en la naturaleza.

La crítica a las concepciones tradicionales del espacio se vio fortalecida por la consolidación de las geometrías no euclídeas, en particular por la demostración de su consistencia. Para finales del siglo ya se contaba con un concepto más amplio acerca del espacio, así como lo que debe entenderse por una geometría.

Esta nueva situación en el mundo de las ideas fue muy bien recibida por físicos y astrónomos, quienes se sentían incómodos con la tradición newtoniana y avanzaban a nuevas posiciones a partir de la teoría de la relatividad. Puede decirse que las geometrías no euclídeas desbrozaron el camino a las modernas teorías físicas ([3, p.368]). 


\section{Referencias}

[1] Bonola, R. (1938) Non-Euclidean geometry. The Open Court Publishing Co., Illinois.

[2] Heath, T.L. (1956) Euclid's Elements. Dover, New York.

[3] Kagán, V.F. (1986) Lobachevski. Mir, Moscú.

[4] Lobachevski, N.I. (1899) Zwei geometrische Abhandlungen. Teubner, Leipzig.

[5] Russell, B. (1956) An essay on the foundations of geometry. Dover, New York.

[6] Rosenfeld, B.A. (1989) A history of non euclidean geometry. Springer-Verlag, New York.

[7] Gray, J. (1989) Ideas of space. Euclidean, non-euclidean and relativistic. Clarendon Press, Oxford. 